\title{
Anisoplanatic Performance of Horizontal-Path Speckle Imaging
}

\author{
C.Carrano
}

This article was submitted to: Advanced Wavefront Control:

Methods, Devices, and Aplications, San Diego, CA 08/03/2003-08/08/2003

U.S. Department of Energy

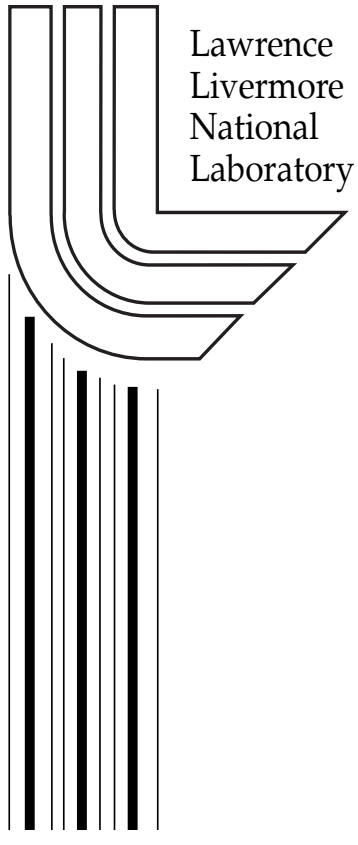

July 1, 2003 
This document was prepared as an account of work sponsored by an agency of the United States Government. Neither the United States Government nor the University of California nor any of their employees, makes any warranty, express or implied, or assumes any legal liability or responsibility for the accuracy, completeness, or usefulness of any information, apparatus, product, or process disclosed, or represents that its use would not infringe privately owned rights. Reference herein to any specific commercial product, process, or service by trade name, trademark, manufacturer, or otherwise, does not necessarily constitute or imply its endorsement, recommendation, or favoring by the United States Government or the University of California. The views and opinions of authors expressed herein do not necessarily state or reflect those of the United States Government or the University of California, and shall not be used for advertising or product endorsement purposes. 


\title{
Anisoplanatic performance of horizontal-path speckle imaging
}

\author{
Carmen J. Carrano* \\ Lawrence Livermore National Laboratory, 7000 East Avenue, Livermore, CA 94551
}

\begin{abstract}
We have previously demonstrated and reported on the use of sub-field speckle processing for the enhancement of both near and far-range surveillance imagery of people and vehicles that have been degraded by atmospheric turbulence ${ }^{1,2,3}$. We have obtained near diffraction-limited imagery in many cases and have shown dramatic image quality improvement in other cases. As it is possible to perform only a limited number of experiments in a limited number of conditions, we have developed a computer simulation capability to aid in the prediction of imaging performance in a wider variation of conditions. Our simulation capability includes the ability to model extended scenes in distributed turbulence. Of great interest is the effect of the isoplanatic angle on speckle imaging performance as well as on single deformable mirror and multiconjugate adaptive optics system performance. These angles are typically quite small over horizontal and slant paths. This paper will begin to explore these issues which are important for predicting the performance of both passive and active horizontal and slant-path imaging systems.
\end{abstract}

Keywords: horizontal-path imaging, slant-path imaging, isoplanatic angle, simulation, bispectral speckle imaging, extended scene reconstruction, long-range surveillance

\section{INTRODUCTION}

High-resolution imaging over long horizontal or slant paths is a difficult task due to atmospheric turbulence. It causes both blurring and warping to various degrees in imagery that is collected over such paths. Speckle imaging techniques seek to eliminate the effects of atmospheric turbulence in images via the phase closure argument ${ }^{4}$. Early in the derivation of speckle imaging theory, the assumption of isoplanatism is made ${ }^{5}$. If two point sources are isoplanatic they undergo the same atmospheric degradation along their optical paths and have the exact same instantaneous point spread function. The isoplanatic angle is defined as the angle between two points at which their mean squared wavefront error due to differences in the atmospheric path is one radian squared ${ }^{6}$. When images of extended scenes are collected over horizontal or slant paths, they are not isoplanatic, because optical paths for different points in the scene do not necessarily see the same atmosphere. In fact, it is quite possible that the isoplanatic angle can be on the order of a few tens of image pixels or less. To help overcome this, we have employed tiling of the image into small regions ${ }^{1}$. The optimal choice of tile size is not obvious, because, as we will show in this paper, it can depend on three primary factors, the isoplanatic angle, the point spread function extent, and the object feature size characteristics. The motivation behind this paper is to explain why we experience significant image improvement using speckle imaging when many isoplanatic angles span a tile.

In this paper we will explore via simulation the effect of isoplanatic angle, PSF extent, and object feature size on speckle image reconstructions. First, we will examine two point sources as they are separated from each other within a given tile. Next, we will examine variably sized checkerboard patterns, which are extended targets with well-defined object size content. Finally, we will examine the image of a face, which is an extended target and relevant to surveillance imaging, in both simulation and actual experiment.

\section{POINT SOURCE SIMULATIONS}

\subsection{Simulation method}

We have developed a capability to simulate speckle imagery of point sources with distributed turbulence. We model the distributed turbulence by splitting the path into atmospheric layers of a certain depth. At the center of each layer we insert a properly sampled Kolmogorov phase screen with a given $r_{0}$ value. Using Fresnel propagation, we propagate

\footnotetext{
*E-mail: carrano2@1lnl.gov
} 
each point source from its origin through each phase screen to the aperture. Once the complex field reaches the aperture, we apply the aperture function, and Fresnel propagate through free space to the image plane position. The intensity images of each point source at the camera are summed to create the multi-point source result. See Figure 1 for an illustration of the procedure. To generate the multiple frames needed for speckle imagery, we repeat the process many times but using newly generated random phase screens each iteration.

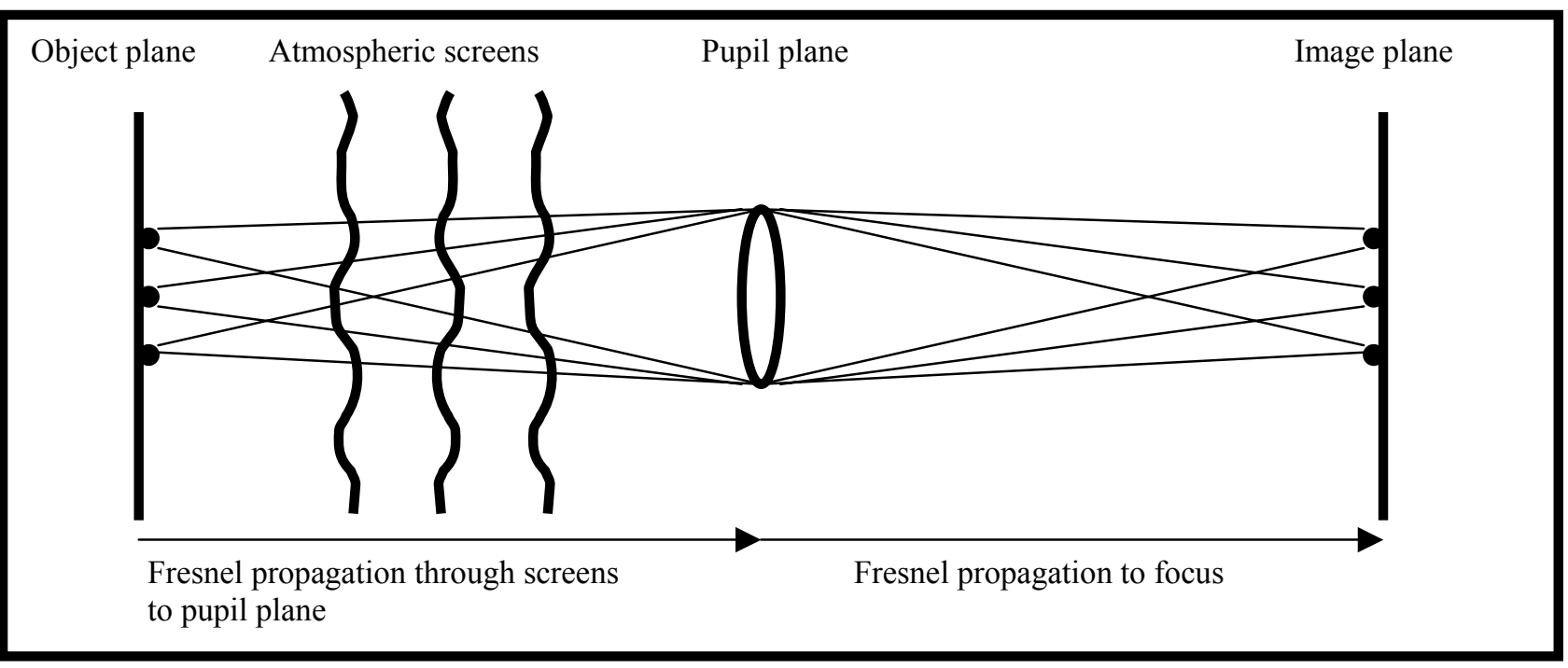

Figure 1: Illustration of distributed turbulence simulation.

For the simulations described in this paper we use a single atmospheric screen at different positions. Although this simplified model clearly does not fully reproduce the effects of a fully distributed horizontal path turbulence, it does give us a controllable anisoplanatism error and allows us to isolate the anisoplanatic and seeing effects. By using a single atmospheric screen, it is possible to vary $\theta_{0}$ as well as the effective $\mathbf{r}_{0}$ over the path in a simple way by varying the placement and the $r_{0}$ of the atmospheric phase screen. Since we have spherical wave propagation, we calculate the effective $r_{0}$ over the path by multiplying the screen $r_{0}$ with the ratio of the object-pupil distance to the object-screen distance. We calculate the isoplanatic angle, $\theta_{0}$, using the simple formula in Equation $1^{6}$.

$$
\theta_{0}=\frac{0.314 r_{0}}{L_{s c r}},
$$

where $\mathrm{L}_{\text {scr }}$ is the screen to pupil distance, and $\mathrm{r}_{0}$ is that of the atmospheric phase screen.

\subsection{Results and Analysis}

For the point source simulations described here, we use a single atmospheric screen placed half way between the object and the pupil and then vary the spacing between the point sources to achieve the anisoplanatic effects. The following parameters were used for the point source simulations:

- Range $\left(\mathrm{L}_{\mathrm{tot}}\right)=1 \mathrm{~km}$

- $\quad$ Telescope Diameter $=20 \mathrm{~cm}, \lambda=0.5 \mu \mathrm{m}$

- Nyquist sampling: $\mathrm{dx}=(\lambda / 2 \mathrm{D}) * \mathrm{~L}_{\mathrm{tot}}=0.125 \mathrm{~cm}$

- Screen location $\left(\mathrm{L}_{\mathrm{scr}}\right)=500 \mathrm{~m}$, Screen $\mathrm{r}_{0}=1.0 \mathrm{~cm}$,

- Effective $\mathrm{r}_{0}=(1000 \mathrm{~m} / 500 \mathrm{~m}) * 1.0 \mathrm{~cm}=2.0 \mathrm{~cm}$

- $\mathrm{D} / \mathrm{r}_{0}=10$

- Isoplanatic patch size at range: $\theta_{0} * \mathrm{~L}_{\text {tot }}=(0.314 * 1.0 \mathrm{~cm} / 500 \mathrm{~m}) * 1000 \mathrm{~m}=0.628 \mathrm{~cm}$

- Point source separation distance ranging from: 5 pixels $=0.625 \mathrm{~cm}=1 \theta_{0} \mathrm{~L}$, up to 100 pixels $=12.5 \mathrm{~cm}=20 \theta_{0} \mathrm{~L}$ 's 
In order to get some statistical variation, a set of 1000 frames was created for each point-source spacing. Twenty sets of fifty frames were then speckle processed with a single 256x256 pixel sized tile. Sample frames and their associated speckle reconstructions are shown in Figure 2 for a $2 \theta_{0} \mathrm{~L}$ separation and a $20 \theta_{0} \mathrm{~L}$ separation. We see that as the point sources are separated, their speckle patterns become less correlated and the processed image degrades in quality somewhat.

We measured the mean and standard deviation of the resulting peak values for each reconstructed point source set and then normalized by the peak value for the no atmosphere case to obtain a Strehl value. In some of the reconstructed images we see that one of the point sources is much brighter than the other while in some reconstructions the points are equally degraded. The variation becomes more apparent and dramatic as their separation is increased. This leads to an effective increase in the variance of the Strehl. We believe this behavior is based on the randomness of the atmospheric screens and their corresponding interaction with the bispectral phase estimation. A plot of the Strehl values with associated error bars is shown in Figure 3. Note that the average Strehl only decreases by about $25 \%$, but the variation of the Strehl values increases significantly. For comparison, a plot of the theoretical performance of a single conjugate adaptive optics (AO) system conjugate to the pupil plane with and without piston, tip and tilt removed is included $6,7,8$. When piston, tip and tilt are removed in a finite aperture telescope the effective isoplanatic angle can increase by a factor of two or more depending on the aperture size ${ }^{8}$. Regardless of the assumptions, the plot in Figure 3 enables us to see how much less sensitive speckle imaging is to isoplanatic angle compared with AO. Of course, a multiple guide-star AO system would exhibit improved performance, likewise, by using multiple tiles the separated point sources suffer minimal or no degradation.

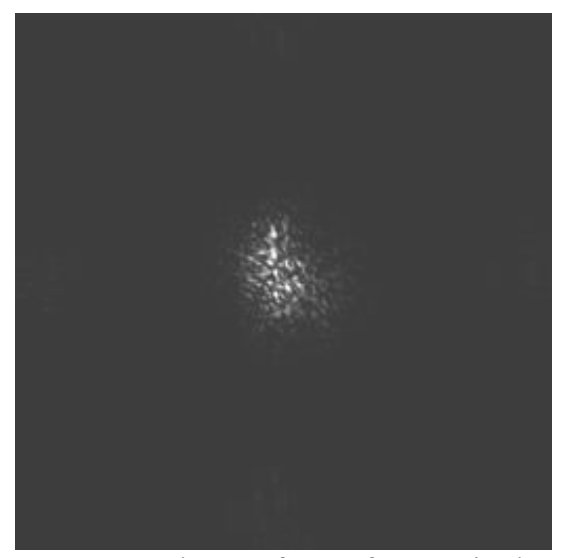

Figure 2a: Sample raw frame for 10 pixel separation

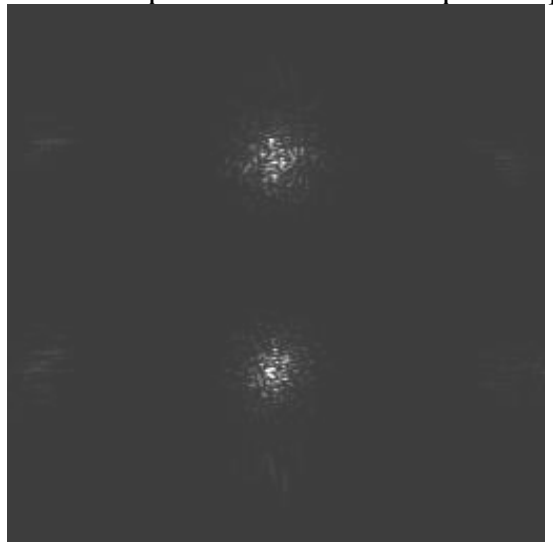

Figure 2c: Sample raw frame for 100 pixel separation

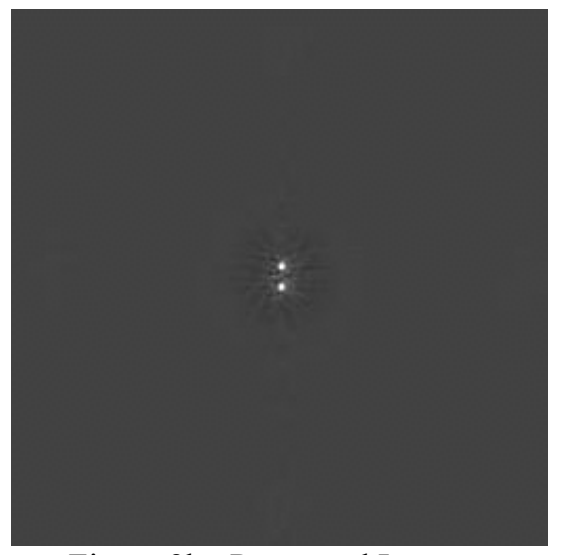

Figure 2b: Processed Image

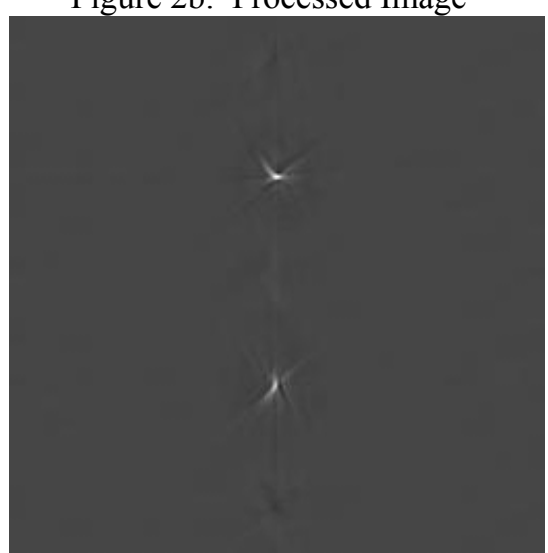

Figure 2d: Processed Image

Figure 2: Examples of sample unprocessed frames for a $2 \theta_{0} \mathrm{~L}$ separation and a $20 \theta_{0} \mathrm{~L}$ separation and their associated processed results using 50 frames. 


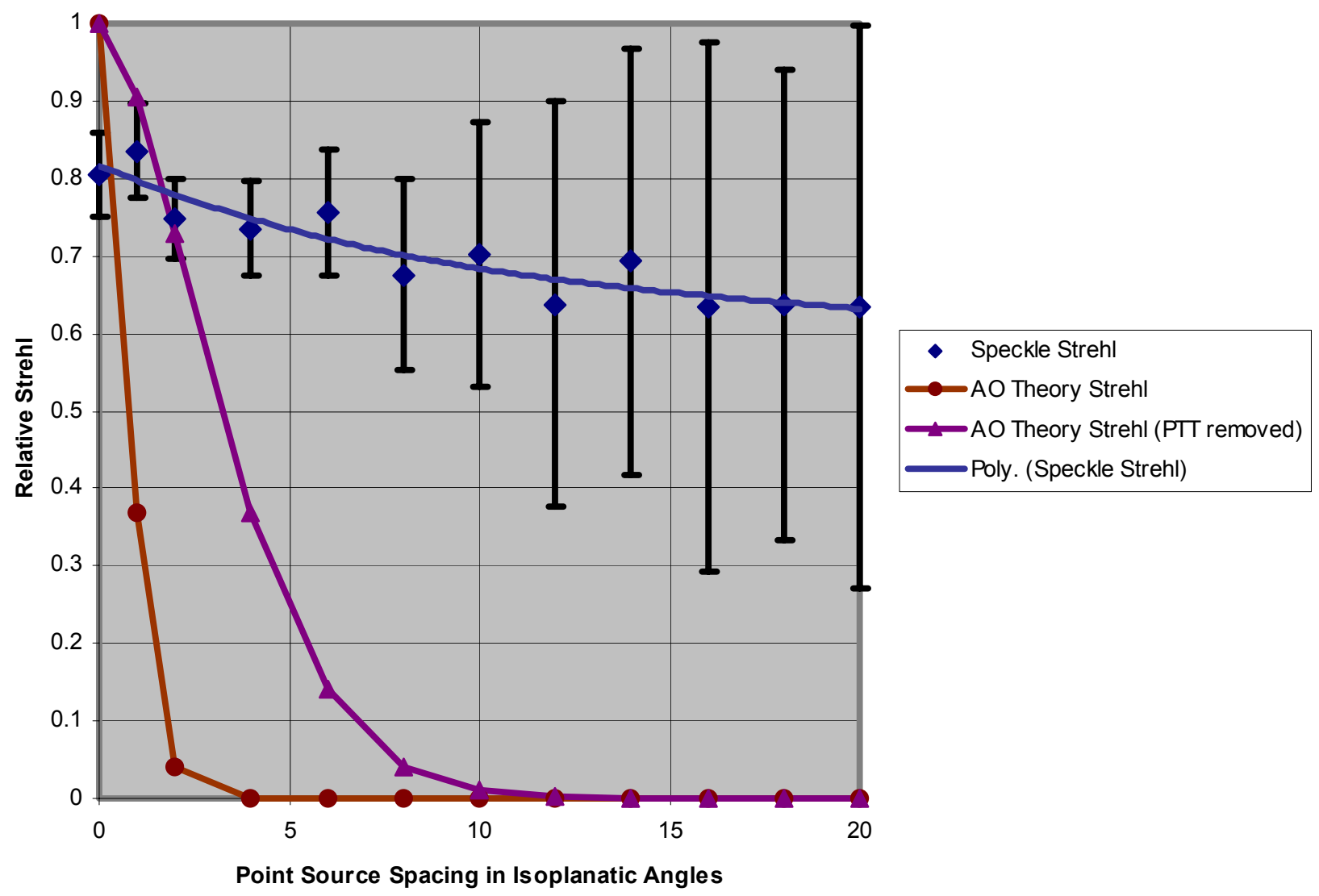

Figure 3: Strehl performance of single tile speckle reconstructions of two point sources as they are spaced further apart. Included are error bars and a $3^{\text {rd }}$ order polynomial fit to the data points. Also plotted for comparison is the theoretical performance of a single conjugate adaptive optics system conjugate to the pupil plane (i.e. Strehl $=\exp \left[-\left(\theta / \theta_{0}\right)^{5 / 3}\right]$ ) with and without piston, tip and tilt removed (i.e. Strehl $\approx \exp \left[\left(-\theta /\left(4 * \theta_{0}\right)\right)^{5 / 3}\right]$ for a small finite aperture $<0.5 \mathrm{~m}$ ).

\section{EXTENDED TARGET SIMULATIONS}

\subsection{Simulation method}

In order to simulate an incoherent extended target of substantial size when the atmosphere is not fully isoplanatic, the method described in Section 2.1 would work, but take far too much time. For example, to propagate every point in the field separately for a $256 \times 256$ pixel sized image requires 65,536 propagations, and for a $512 \times 512$ pixel sized image requires 262,144 propagations. We have developed a random phase method that requires less than 10,000 propagations. First, a complex field at the object plane is constructed which consists of the magnitude of the object and a uniformly distributed random phase over 0 to $2 \pi$. Then the field is propagated in its entirety through the atmospheric screens and an image is formed. This is repeated with new random object phase screens to form more realizations of the image. The resulting intensity images from these randomly phased object propagations are then added together. To create a single speckle image frame, it takes several thousand of these realizations to reduce the noise sufficiently introduced by the random phase. The block diagram in Figure 4 illustrates the simulation sequence. To generate multiple speckle images, a new set of atmospheric screens is created and the sequence is repeated.

We typically use between 5000 and 8000 realizations for each screen in our simulations. We have implemented the software on a 512 processor parallel computer system at LLNL. Each of the Compaq ES40 EV67 processors runs at 
$667 \mathrm{MHz}$ with a combined potential peak performance of $681 \mathrm{Gflop} / \mathrm{s}$. Using 48 processors, it currently takes about 9 minutes to generate each $512 \times 512$ sized speckle image frame using a single atmospheric screen with 8000 realizations. This means to generate 50 frames of data takes nearly 8 hours.

To better illustrate the random phase simulation method, the following set of images in Figure 5 shows the no atmosphere case with increasing numbers of realizations. As we see in the images, the noise is significantly reduced after 1000 realizations.

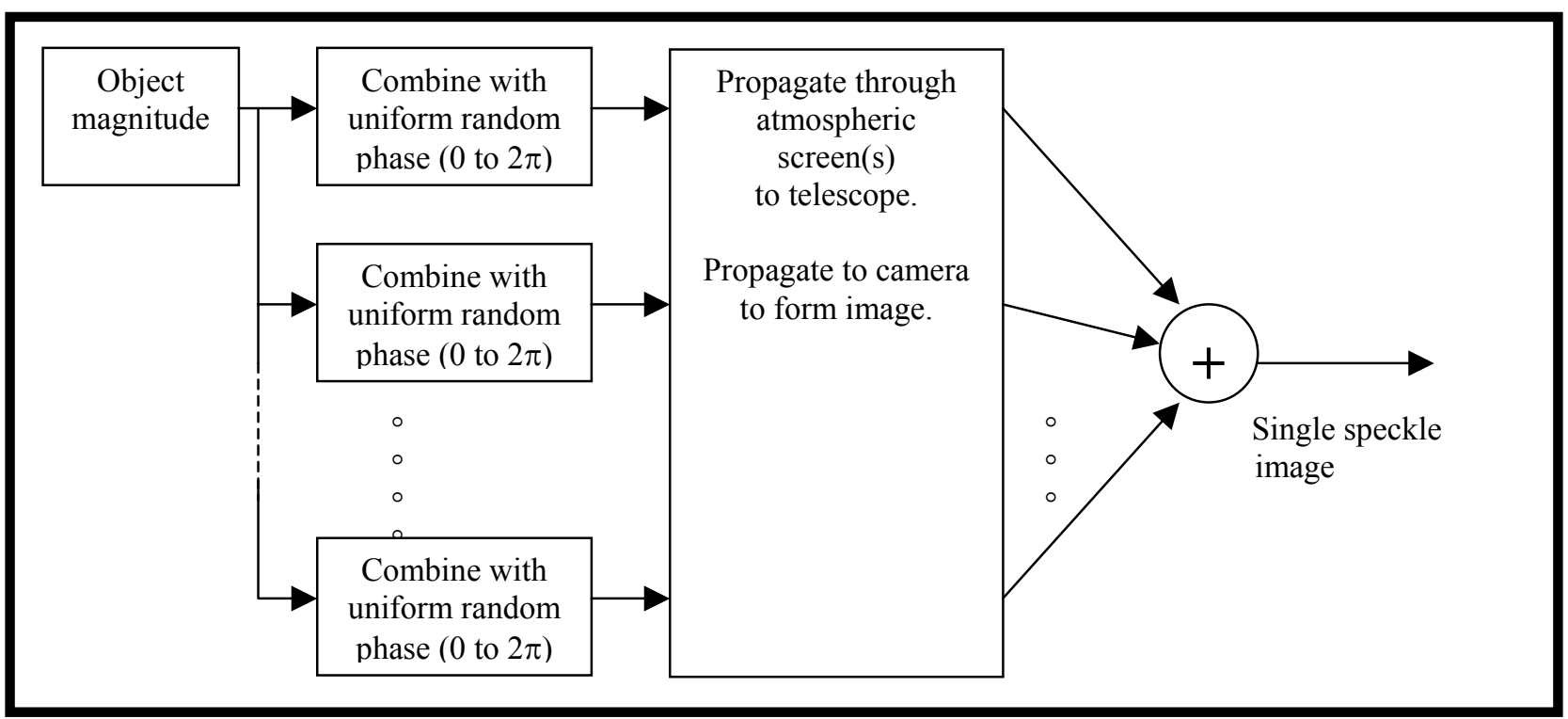

Figure 4: Block diagram of random phase simulation method for extended incoherent objects with distributed turbulence.

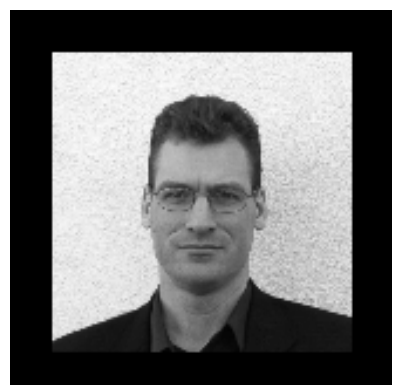

5a. Original object

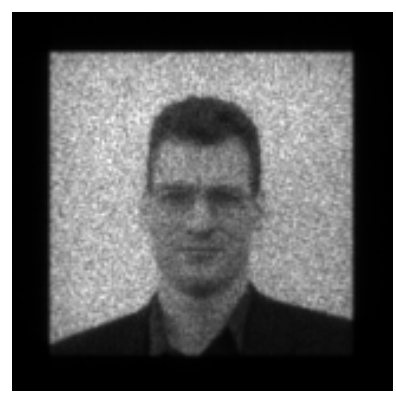

5d. 100 realizations

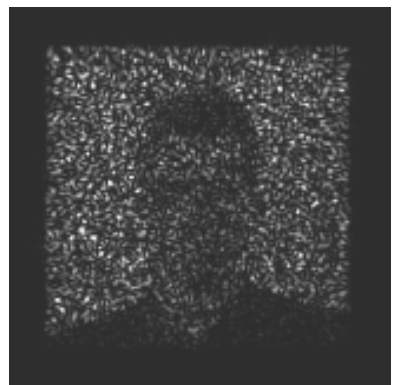

5b. Single realization

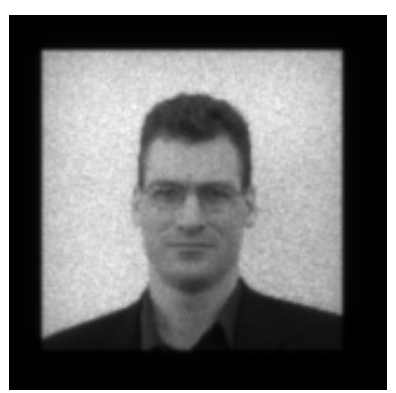

5e. 1,000 realizations

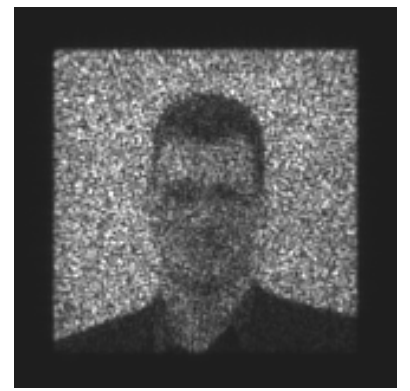

5c. 10 realizations

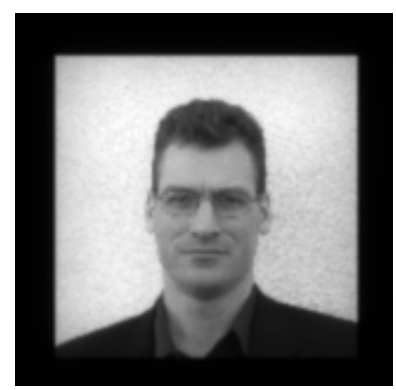

5f. 10,000 realizations

Figure 5: Illustration of the dependence of the resulting image quality versus the number of random realizations for an extended object with no atmosphere. 


\subsection{Checkerboard simulation results}

The checkerboard pattern is an object that will allow clear demonstration of the dependence of processing tile size on object feature size characteristics as well as isoplanatic angle and PSF extent. We have performed simulations of checkerboard patterns of varying checker size and isoplanatic angle to illustrate this.

The following parameters are common throughout the checkerboard simulations:

- $\quad$ Range $\left(\mathrm{L}_{\text {tot }}\right)=1 \mathrm{~km}$

- $\quad$ Telescope Diameter $=20 \mathrm{~cm}, \lambda=0.5 \mu \mathrm{m}$

- Nyquist sampling: $\mathrm{dx}=(\lambda / 2 \mathrm{D}) * \mathrm{~L}_{\text {tot }}=0.125 \mathrm{~cm} /$ pixel

- 8000 random realizations per speckle frame with 50 speckle frames per reconstruction

The first set of checkerboard simulations have a 60 pixel $\left(\right.$ or $7.5 \mathrm{~cm}$ ) wide checker size and a $\mathrm{D} / \mathrm{r}_{0}$ of 10 . A single atmospheric screen is placed halfway between the telescope and the object at 500 meters with an $\mathrm{r}_{0}$ of $1.0 \mathrm{~cm}$ giving a projected isoplanatic patch size of $0.63 \mathrm{~cm}$ on the object. The images shown in Figure 6 clearly illustrate that the $128 \times 128$ tile size reconstruction does give the best results, though the $256 \times 256$ case is not far behind. An important item to note is the number of isoplanatic angles across the active tile, listed in Figure $6 \mathrm{f}$. The active tile size is calculated as $60 \%$ of the full tile size, since we are using $60 \%$ apodization in the processing. Although there are far fewer isoplanatic angles across them, the smaller tiles give worse results for several reasons. One, the tile is clipping the PSF, especially in the $32 \times 32$ case, which means that not all the possible and needed information is being captured for even the central point in the tile. We know this because for a $\mathrm{D} / \mathrm{r} 0$ of 10 sampled at Nyquist (i.e. such that there are 2 pixels across a diffraction limited peak), the full width half maximum of the PSF should be approximately 20 pixels with the tails extending well beyond that. A $32 \times 32$ window with $60 \%$ apodization only spans 19 pixels before it begins to fall to zero. And two, the apodization window edges are steeper than the blurring induced by the point spread function, even starting with the $64 \times 64$ tile case, which contributes to tiling artifacts. Additionally, it is beneficial for the reconstruction that the $128 \times 128$ window can completely isolate each one of the checkers while the other sized tiles do not.

The next set of simulation results has the same $\mathrm{D} / \mathrm{r}_{0}$ of 10 and differs from the previous only by the isoplanatic angle. Using an atmospheric screen at 250 meters from the object with an $\mathrm{r}_{0}$ of $0.5 \mathrm{~cm}$ yields a projected isoplanatic patch size of $0.21 \mathrm{~cm}$ on the object. We display the results in Figure 7. Note that in this case the 256x256 tile sized result, Figure $7 \mathrm{a}$, is degraded significantly from the previous case, Figure $6 \mathrm{a}$. This is because there are three times as many isoplanatic angles across the tile as before. Likewise, the 128x128 tile sized result in Figure 7c is degraded from Figure $6 \mathrm{c}$ due to increased isoplanatic angles across its tile, but it remains the best result. A close second is the $64 \times 64$ size tile, though the interaction between the tile and the PSF are noticeable.

The next two sets of simulation results illustrate what happens when the object feature size is cut in half to 30 pixels or $3.75 \mathrm{~cm}$. They possess the same setup as the previous two simulations. Figure 8 shows the $0.63 \mathrm{~cm}$ isoplanatic patch size case and Figure 9 shows the $0.21 \mathrm{~cm}$ isoplanatic patch size case. For the larger isoplanatic angle case, the $128 \times 128$ pixel sized tile performs the best, with the $64 \times 64$ pixel sized tile a close second. But for the smaller isoplanatic angle case, unlike the 60 pixel checker size, we find that for the 30 pixel checker size, the $64 x 64$ pixel tile performs the best in part because it has the best match to the checker size although it has some tiling window-PSF interaction artifacts,

In order to demonstrate where the $64 \times 64$ tile window can be useful, we show a final checkerboard example with a $\mathrm{D} / \mathrm{r}_{0}$ of 5 , but still with a $0.22 \mathrm{~cm}$ projected isoplanatic patch size at the object. By decreasing the $\mathrm{D} / \mathrm{r}_{0}$ we are decreasing the size of the atmospheric point spread function, hence steepening the slope of the blur induced on an edge by the PSF. Because it is steeper than the tile window apodization, the $64 \times 64$ tile sized window does not interfere and is able to operate free from artifacts. The results are displayed in Figure 10. Notice that the 32x32 tile window case has improved significantly due to less PSF clipping.

Based on the collective results from the checkerboard patterns, we can make some general observations about how sensitive extended scene speckle imaging is to isoplanatic angle. For a typical case with a $\mathrm{D} / \mathrm{r}_{0}$ of 10 , it appears that upwards of 20 to 30 isoplanatic angles can span a tile to obtain very good reconstructions, regardless of the object content, while upwards of 45 isoplanatic angles can be handled to a lesser degree when there is a match between the tile size and the characteristic object size. Better atmosphere (i.e. smaller $\mathrm{D} / \mathrm{r}_{0}$ ) allows the use of smaller tiles if they are needed. 


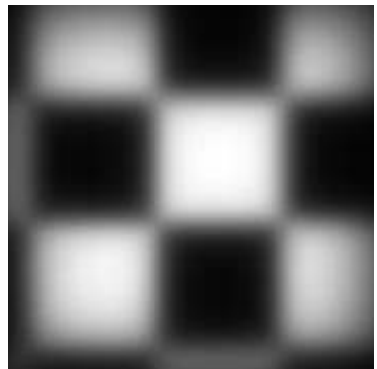

6a. Shift and add 50 frames

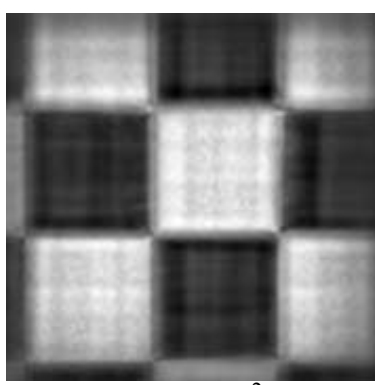

6d. Processed with $64^{2}$ sized tiles

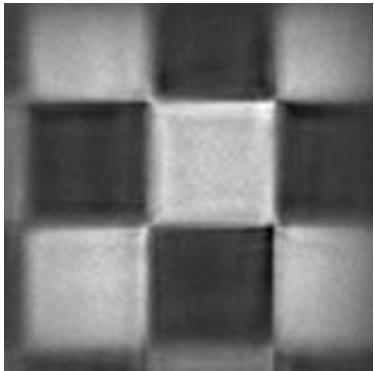

6b. Processed with a $256^{2}$ sized tile

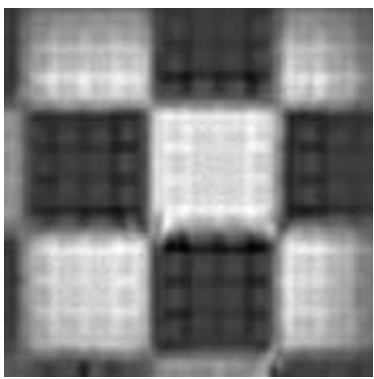

6e. Processed with $32^{2}$ sized tiles

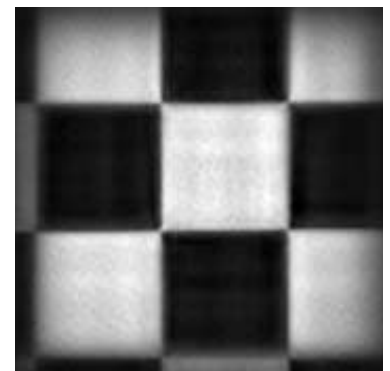

6c. Processed with $128^{2}$ sized tiles

\begin{tabular}{|l|c|}
\hline $\begin{array}{l}\text { Tile size } \\
\text { (pixels) }\end{array}$ & $\begin{array}{c}\text { Isoplanatic angles } \\
\text { across tile }\end{array}$ \\
\hline $256 \times 256$ & 30.6 \\
$128 \times 128$ & 15.2 \\
$64 \times 64$ & 7.6 \\
$32 \times 32$ & 3.8 \\
\hline
\end{tabular}

6f. Table of the number of isoplanatic angles across each active tile.

Figure 6: Speckle processing results for a 60 pixel checker size checkerboard pattern as the tile size is changed, where $\mathrm{D} / \mathrm{r}_{0}=10$ and $\theta_{0} * \mathrm{~L}=0.63 \mathrm{~cm}$.

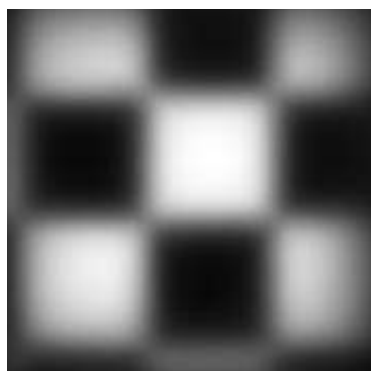

7a. Shift and add 50 frames

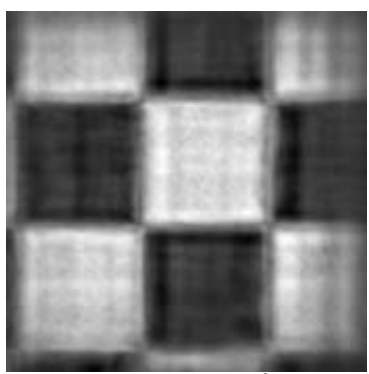

7d. Processed with $64^{2}$ sized tiles

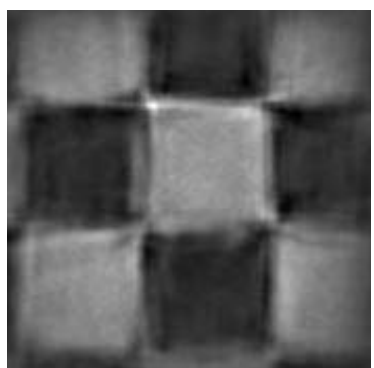

7b. Processed with a $256^{2}$ sized tile

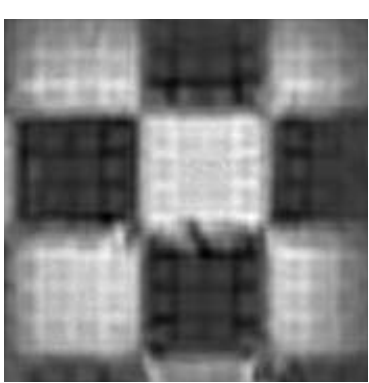

7e. Processed with $32^{2}$ sized tiles

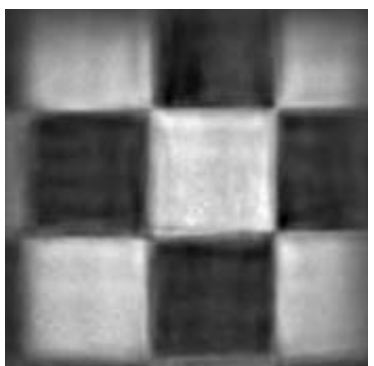

7c. Processed with $128^{2}$ sized tiles

\begin{tabular}{|l|c|}
\hline $\begin{array}{l}\text { Tile size } \\
\text { (pixels) }\end{array}$ & $\begin{array}{c}\text { Isoplanatic angles } \\
\text { across tile }\end{array}$ \\
\hline $256 \times 256$ & 91.4 \\
$128 \times 128$ & 45.7 \\
$64 \times 64$ & 22.8 \\
$32 \times 32$ & 11.4 \\
\hline
\end{tabular}

7f. Table of the number of isoplanatic angles across each active tile.

Figure 7: Speckle processing results for a 60 pixel checker size checkerboard pattern as the tile size is changed, where $\mathrm{D} / \mathrm{r}_{0}=10$ and $\theta_{0} * \mathrm{~L}=0.21 \mathrm{~cm}$. 


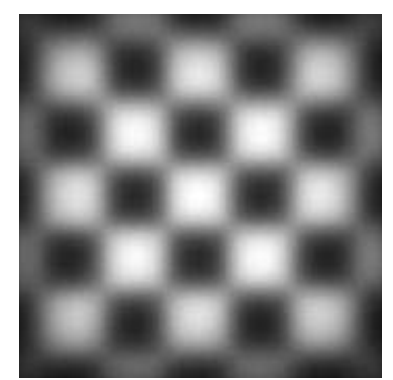

8a. Shift and add 50 frames

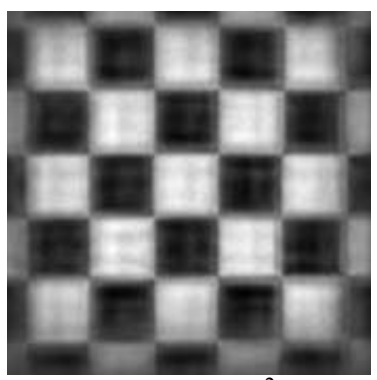

8d. Processed with $64^{2}$ sized tiles

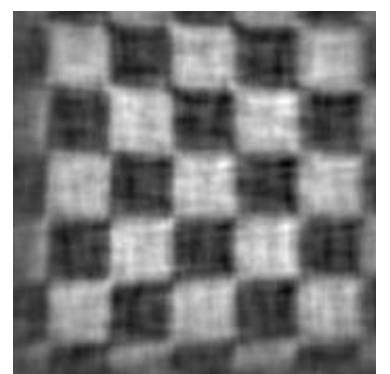

8 b. Processed with a $256^{2}$ sized tile

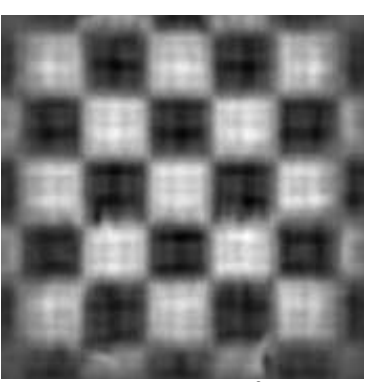

8e. Processed with $32^{2}$ sized tiles

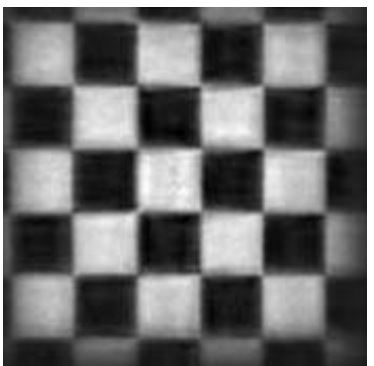

8c. Processed with $128^{2}$ sized tiles

\begin{tabular}{|l|c|}
\hline $\begin{array}{l}\text { Tile size } \\
\text { (pixels) }\end{array}$ & $\begin{array}{c}\text { Isoplanatic angles } \\
\text { across tile }\end{array}$ \\
\hline $256 \times 256$ & 30.6 \\
$128 \times 128$ & 15.2 \\
$64 \times 64$ & 7.6 \\
$32 \times 32$ & 3.8 \\
\hline
\end{tabular}

$8 \mathrm{f}$. Table of the number of isoplanatic angles across each active tile.

Figure 8: Speckle processing results for a 30 pixel checker size checkerboard pattern as the tile size is changed, where $\mathrm{D} / \mathrm{r}_{0}=10$ and $\theta_{0} * \mathrm{~L}=0.63 \mathrm{~cm}$.

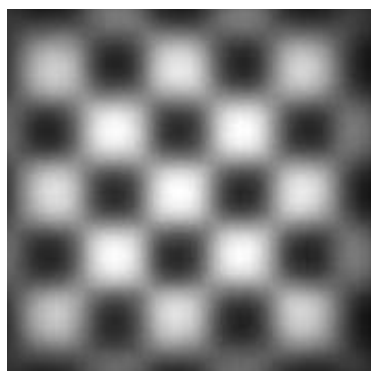

9a. Shift and add 50 frames

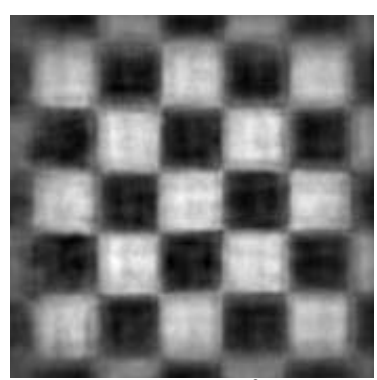

9d. Processed with $64^{2}$ sized tiles

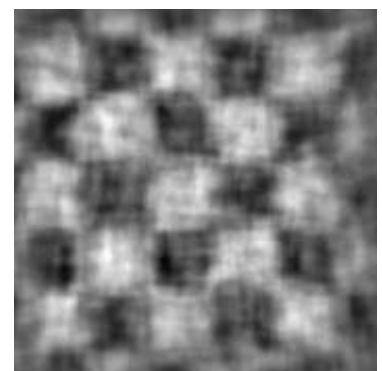

9b. Processed with a $256^{2}$ sized tile

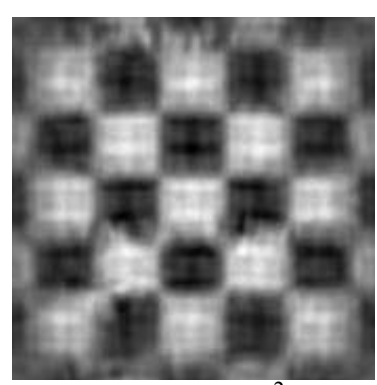

9e. Processed with $32^{2}$ sized tiles

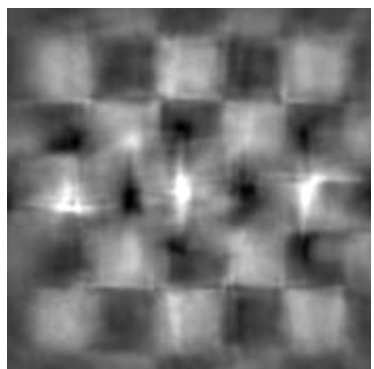

9c. Processed with $128^{2}$ sized tiles

\begin{tabular}{|l|c|}
\hline $\begin{array}{l}\text { Tile size } \\
\text { (pixels) }\end{array}$ & $\begin{array}{c}\text { Isoplanatic angles } \\
\text { across tile }\end{array}$ \\
\hline $256 \times 256$ & 91.4 \\
$128 \times 128$ & 45.7 \\
$64 \times 64$ & 22.8 \\
$32 \times 32$ & 11.4 \\
\hline
\end{tabular}

9f. Table of the number of isoplanatic angles across each active tile.

Figure 9: Speckle processing results for a 30 pixel checker size checkerboard pattern as the tile size is changed, where $\mathrm{D} / \mathrm{r}_{0}=10$ and $\theta_{0}^{*} \mathrm{~L}=0.21 \mathrm{~cm}$. 


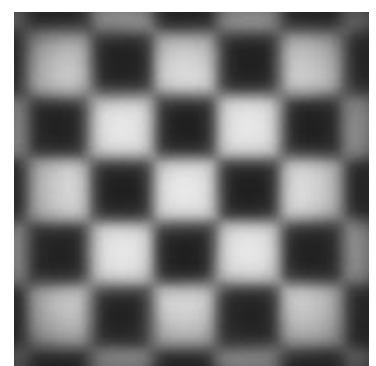

10a. Shift and add 50 frames

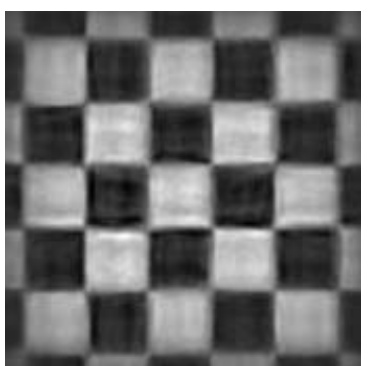

10b. Processed with a $64^{2}$ sized tile

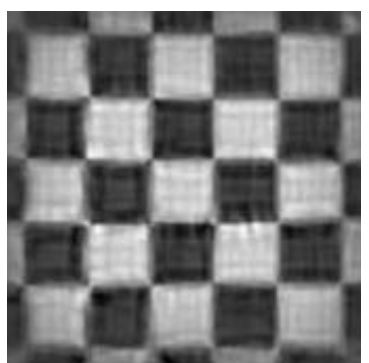

10c. Processed with $32^{2}$ sized tiles

Figure 10: Shift and add and speckle processing results for a 30 pixel checker size checkerboard pattern for $64^{2}$ and $32^{2}$ pixel sized tiles, where $\mathrm{D} / \mathrm{r}_{0}=5$ and $\theta_{0} * \mathrm{~L}=0.22 \mathrm{~cm}$.

\subsection{Face simulation results}

We now move on to a face target which has more relevance to surveillance imaging. A photograph of our subject was obtained and then resampled to $0.125 \mathrm{~cm} /$ pixel so it could be used as the input object.

Just as in the checkerboard simulations, the following parameters are common throughout the face simulations:

- Range $\left(\mathrm{L}_{\mathrm{tot}}\right)=1 \mathrm{~km}$

- Telescope Diameter $=20 \mathrm{~cm}, \lambda=0.5 \mu \mathrm{m}$

- Nyquist sampling: $\mathrm{dx}=(\lambda / 2 \mathrm{D}) * \mathrm{~L}_{\text {tot }}=0.125 \mathrm{~cm} /$ pixel

- 8000 random realizations per speckle frame with 50 speckle frames per reconstruction

Figures 11,12 , and 13 have a common $\mathrm{D} / \mathrm{r}_{0}$ of 10 , while the isoplanatic patch size is being decreased from a $0.628 \mathrm{~cm}$ at the object, to $0.38 \mathrm{~cm}$, and finally to $0.21 \mathrm{~cm}$. We see from the sample frames for each case that the raw data becomes more distorted as isoplanatic angle decreases. As for the speckle reconstructions, in Figure 11, the 128x128 sized tile performs the best, with the $256 \times 256$ sized tile reconstruction coming in a close second. The $64 \times 64$ sized tile case is experiencing problems with artifacts, just as in the checkerboard case, because of PSF clipping and the tile window apodization is steeper than the blur induced by the atmosphere. In Figure 12, the 256x256 sized tile reconstuction is now suffering considerable degradation due to isoplanatism, while the $128 \times 128$ sized tile case still reconstructs quite well. The 64x64 sized tile case continues to exhibit tile-window/PSF interaction effects. Now when we decrease the isoplanatic angle below the diffraction limit, as in Figure 13, the 256x256 sized tile result is unrecognizable. While the $128 \times 128$ sized tile result suffers from noticeable distortion, it is still an improvement over the raw frames or the stabilized image. The $64 \times 64$ case exhibits less distortion of the object, but remains plagued by tile-window/PSF interaction effects.

As performed for the checkerboards, in order to demonstrate when a $64 \times 64$ sized tile window can be of use, we performed a simulation with a $\mathrm{D} / \mathrm{r}_{0}$ of 5 , but still with a $0.22 \mathrm{~cm}$ projected isoplanatic patch size at the object. The simulation results are shown in Figure 14. The 64x64 result is a more correct representation of what the face looks like than the $128 \times 128$ case and there are minimal tile-window/PSF interaction artifacts. 


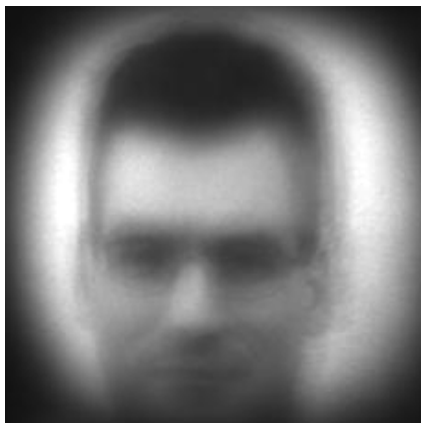

11a. Sample frame

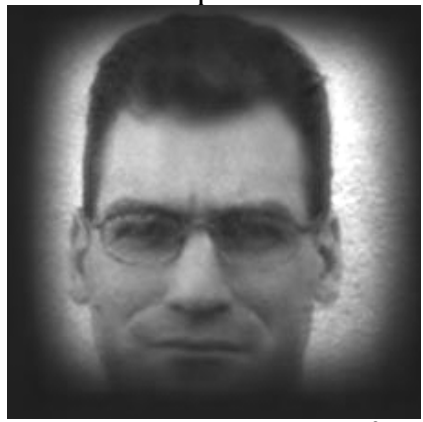

11d. Speckle processing $128^{2}$ tile

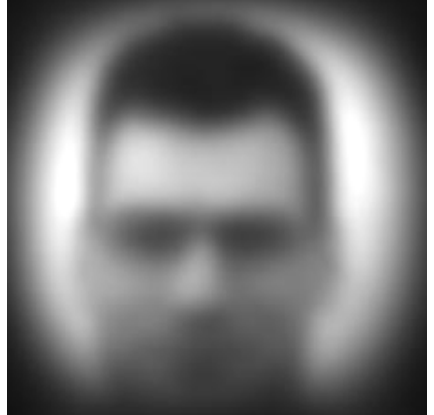

11b. Shift and add

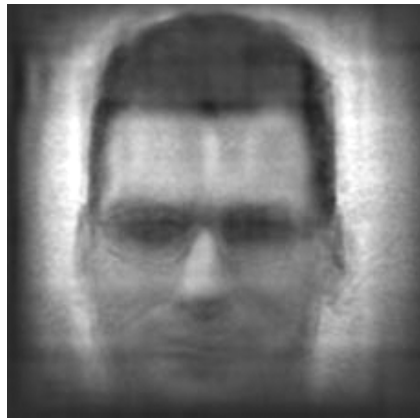

11e. Speckle processing $64^{2}$ tile

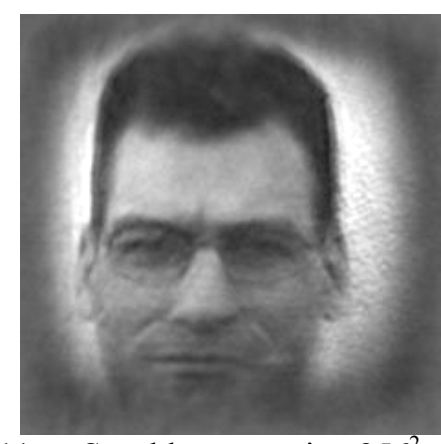

11c. Speckle processing $256^{2}$ tile

\begin{tabular}{|l|c|}
\hline $\begin{array}{l}\text { Tile size } \\
\text { (pixels) }\end{array}$ & $\begin{array}{c}\text { Isoplanatic angles } \\
\text { across tile }\end{array}$ \\
\hline $256 \times 256$ & 30.6 \\
$128 \times 128$ & 15.2 \\
$64 \times 64$ & 7.6 \\
\hline
\end{tabular}

11f. Table of tile size vs. $\theta_{0}$ 's per tile

Figure 11: Sample frame, shift and add, and speckle processing results for a face as the tile size is changed, where $\mathrm{D} / \mathrm{r}_{0}=10$ and $\theta_{0} * \mathrm{~L}=0.628 \mathrm{~cm}$.

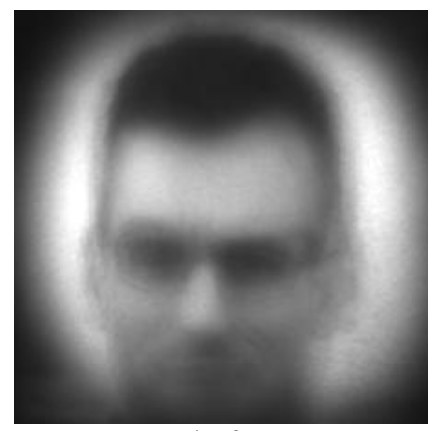

12a. Sample frame

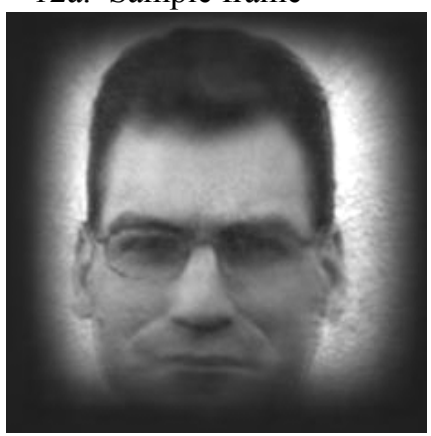

12d. Speckle processing $128^{2}$ tile
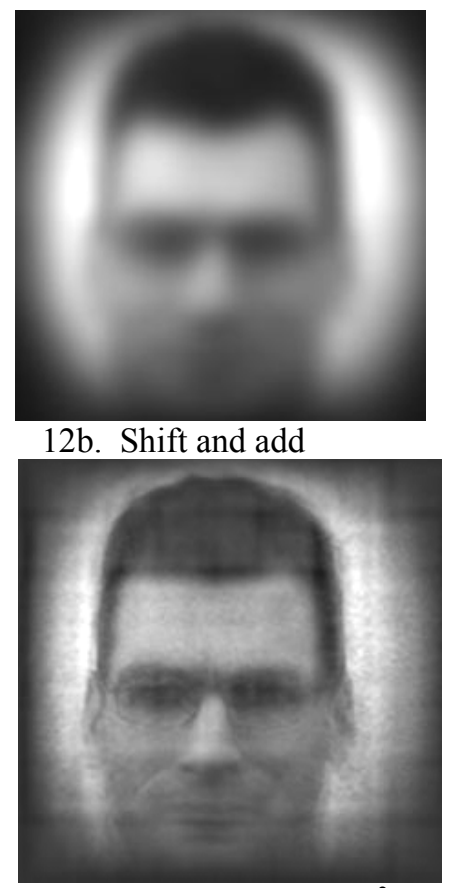

12e. Speckle processing $64^{2}$ tile

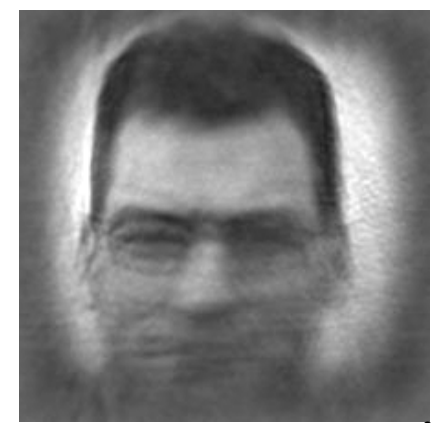

12c. Speckle processing $256^{2}$ tile

\begin{tabular}{|l|c|}
\hline $\begin{array}{l}\text { Tile size } \\
\text { (pixels) }\end{array}$ & $\begin{array}{c}\text { Isoplanatic angles } \\
\text { across tile }\end{array}$ \\
\hline $256 \times 256$ & 50 \\
$128 \times 128$ & 25 \\
$64 \times 64$ & 12.5 \\
\hline
\end{tabular}

12f. Table of tile size vs. $\theta_{0}$ 's per tile

Figure 12: Sample frame, shift and add, and speckle processing results for a face as the tile size is changed, where $\mathrm{D} / \mathrm{r}_{0}=10$ and $\theta_{0} * \mathrm{~L}=0.38 \mathrm{~cm}$. 


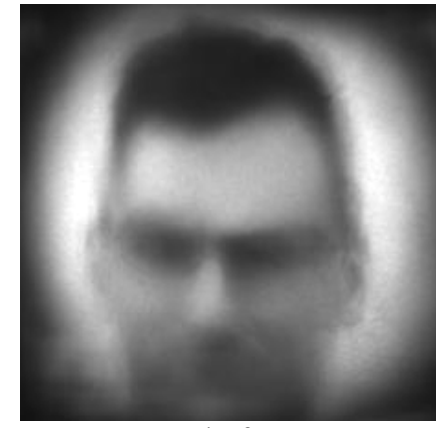

12a. Sample frame

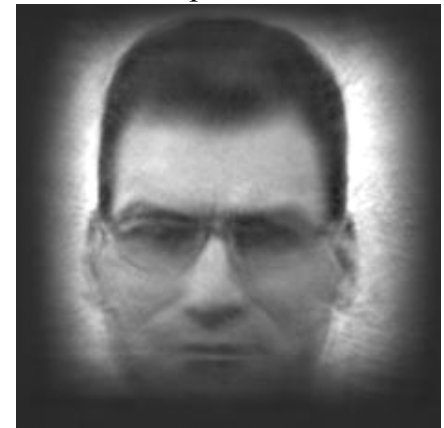

12d. Speckle processing $128^{2}$ tile

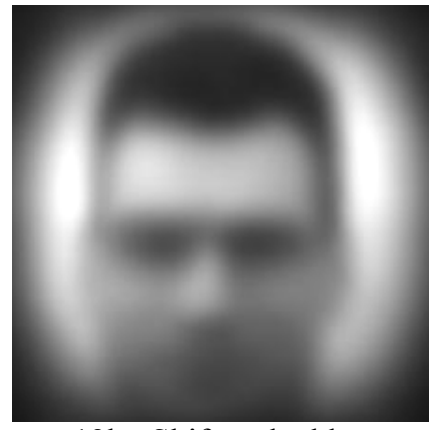

12b. Shift and add

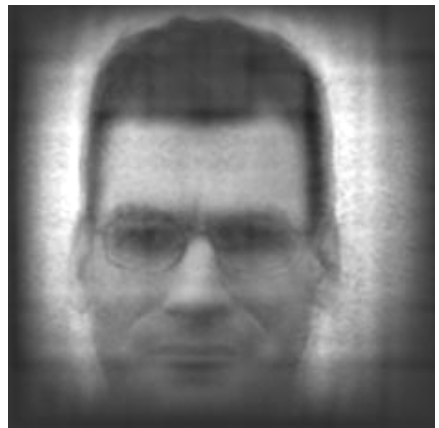

12e. Speckle processing $64^{2}$ tile

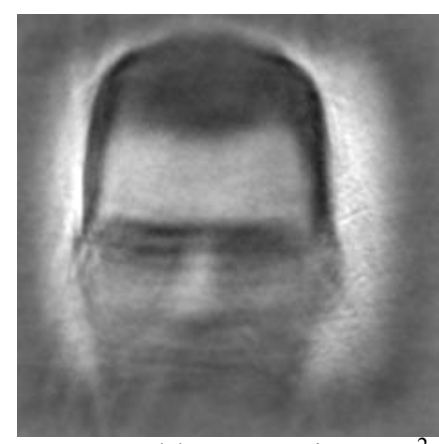

12c. Speckle processing $256^{2}$ tile

\begin{tabular}{|l|c|}
\hline $\begin{array}{l}\text { Tile size } \\
\text { (pixels) }\end{array}$ & $\begin{array}{c}\text { Isoplanatic angles } \\
\text { across tile }\end{array}$ \\
\hline $256 \times 256$ & 91.4 \\
$128 \times 128$ & 45.7 \\
$64 \times 64$ & 22.8 \\
\hline
\end{tabular}

12f. Table of tile size vs. $\theta_{0}$ 's per tile

Figure 12: Sample frame, shift and add, and speckle processing results for a face as the tile size is changed, where $\mathrm{D} / \mathrm{r}_{0}=10$ and $\theta_{0} * \mathrm{~L}=0.21 \mathrm{~cm}$.

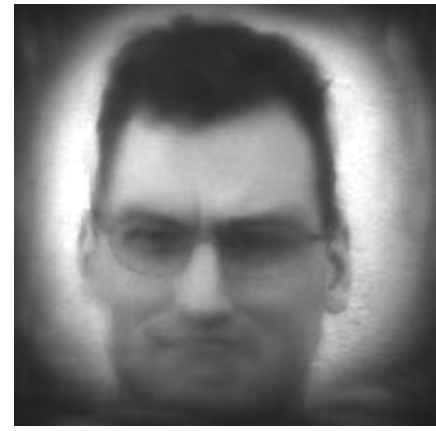

13a. Sample frame

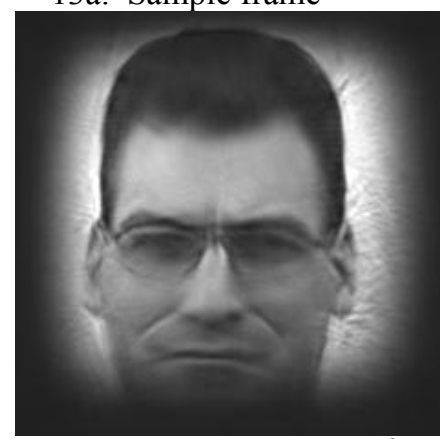

13d. Speckle processing $128^{2}$ tile

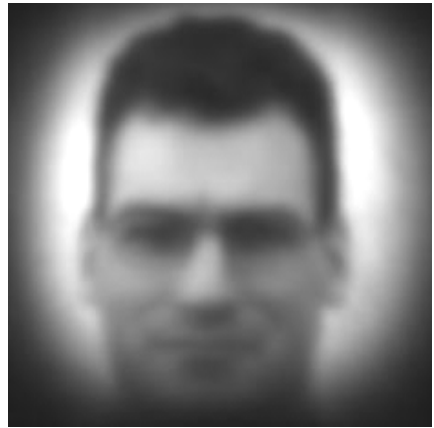

13b. Shift and add

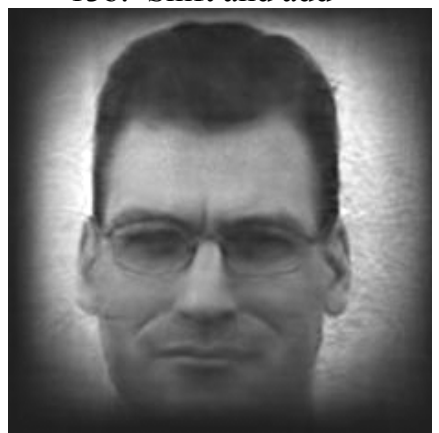

13e. Speckle processing $64^{2}$ tile

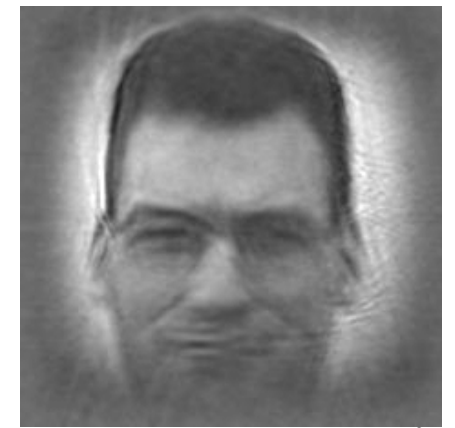

13c. Speckle processing $256^{2}$ tile

\begin{tabular}{|l|c|}
\hline $\begin{array}{l}\text { Tile size } \\
\text { (pixels) }\end{array}$ & $\begin{array}{c}\text { Isoplanatic angles } \\
\text { across tile }\end{array}$ \\
\hline $256 \times 256$ & 87.4 \\
$128 \times 128$ & 43.6 \\
$64 \times 64$ & 21.8 \\
\hline
\end{tabular}

13f. Table of tile size vs. $\theta_{0}$ 's per tile

Figure 13: Sample frame, shift and add, and speckle processing results for a face as the tile size is changed, where $\mathrm{D} / \mathrm{r}_{0}=5$ and $\theta_{0} * \mathrm{~L}=0.22 \mathrm{~cm}$ 


\section{EXPERIMENTAL DATA CHECK}

Now consider a recent experiment that we performed over a horizontal path of $1 \mathrm{~km}$. The estimated $\mathrm{r}_{0}$ for this dataset is $1.7 \mathrm{~cm}$. Since our telescope has a $20 \mathrm{~cm}$ diameter, $\mathrm{D} / \mathrm{r}_{0}$ is 11.7 . Using the equations ${ }^{8}$ for a situation where $\mathrm{C}_{\mathrm{n}}{ }^{2}$ is constant and spherical wave propagations, we estimate $\mathrm{C}_{\mathrm{n}}^{2}$ to be $3.56 \times 10^{-14}$, where

$$
C_{n}{ }^{2}=\frac{0.16 r_{0}^{-5 / 3} \lambda^{2}}{L}
$$

and the isoplanatic angle is calculated to be $5.33 \times 10^{-6}$ radians, where

$$
\theta_{0}=\left(1.093 k^{2} C_{n}^{2} L^{8 / 3}\right)^{-3 / 5}
$$

The sample interval of the data is $1.12 \times 10^{-6}$ radians/pixel. This means there are only 4.8 pixels per isoplanatic angle and approximately 32 isoplanatic angles across an active 256x256 pixel size tile and 16 for a 128x128 sized tile. The results displayed in Figure 14 verify that a 256x256 tile window gives a nice reconstructed result with some remaining blur, especially around the nose and mouth area. The 128x128 sized tile image gives better clarity on the nose and mouth region and well as the badge hanging on his right shoulder. The ear and surrounding hair doesn't look quite as clear as in the $256 \times 256$ case. Sometimes, the details of how the tiles line up with respect to the object feature can affect the outcome slightly. Also, there is another factor in the real data, not explored in the simulations and beyond the scope of this paper, which is the fact that there is both read noise and photon noise to contend with. This experimental case is most similar to the simulation case examined in Figure 11.
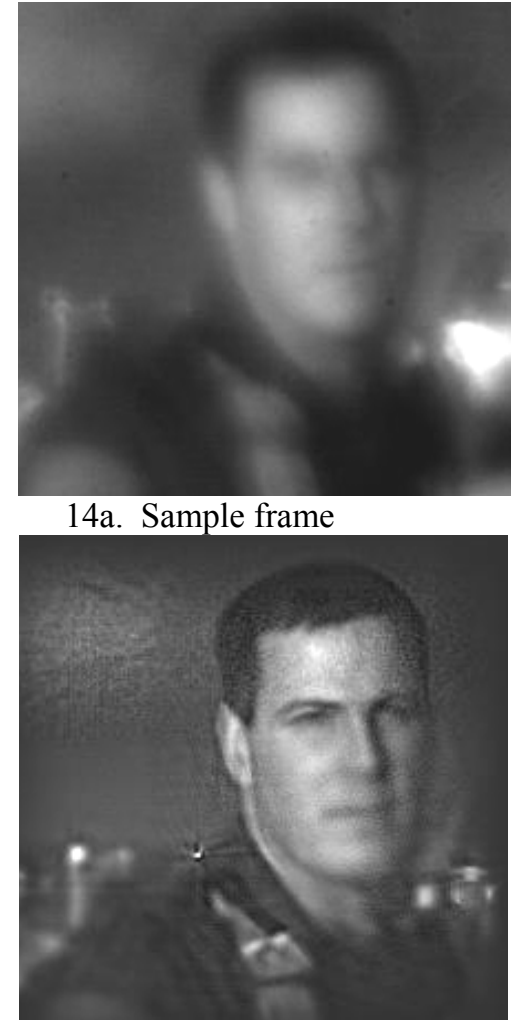

14c. Processed with $256^{2}$ size tiles

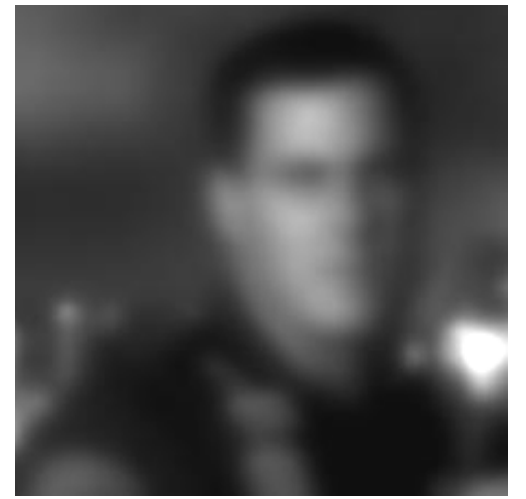

14b. Shift and add 100 frames

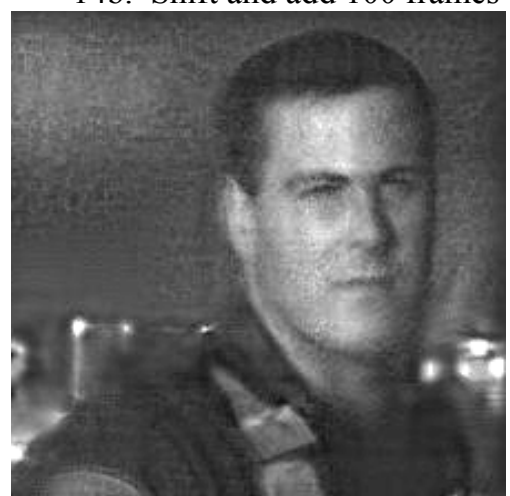

14d. Processed with $128^{2}$ sized tiles

Figure 14: Sample frame, shift and add, and speckle processed results of a face at $1 \mathrm{~km}$ over a horizontal path with an estimated $\mathrm{D} / \mathrm{r} 0$ of 11.7 and an isoplanatic patch size just $\sim 5$ pixels on the image using two different tile sizes. 


\section{CONCLUSIONS}

Based on results presented in this paper, we can make several conclusions about extended scene speckle imaging using tiling methods. Speckle imaging is much less sensitive to isoplanatic angle than traditional single conjugate adaptive optics. Even so, as the isoplanatic angle decreases, the needed tile size to obtain distortion free results decreases. But, as $\mathrm{r}_{0}$ decreases, the necessary tile size to avoid PSF interaction effects and artifacts increases directly proportional to the PSF extent and slope. For most types of imaging paths, such as horizontal or low slant paths, isoplanatic angle decreases with $\mathrm{r}_{0}$. The point where these two lines meet determines the regime where speckle imaging will decrease in effectiveness. Luckily, this regime is limited to quite extreme atmospheric cases and a possible solution to obtain better results is to image using longer wavelengths. We can actually estimate this transition point by using the following assumptions:

$$
\begin{aligned}
& \text { ActiveTilesize(radians) } \geq 3 \frac{\lambda}{r_{0}} \\
& \text { ActiveTilesize(radians) } \leq 30 \theta_{0}
\end{aligned}
$$

If we assume a horizontal path case ( $\mathrm{L}=$ total path length, $\mathrm{r}_{0}$ is over the total path), or alternatively, the simple single atmospheric layer case ( $\mathrm{L}=$ telescope to atmospheric layer distance, $\mathrm{r}_{0}$ is at the layer), we can again use the relationship,

$$
\theta_{0}=\frac{0.314 r_{0}}{L}
$$

and set the inequality,

$$
3 \frac{\lambda}{r_{0}} \leq 30\left[\frac{0.314 r_{0}}{L}\right]
$$

we obtain,

$$
r_{0} \geq \sqrt{0.318 \lambda L}
$$

as the needed $\mathrm{r}_{0}$ for speckle imaging to give the best results.

Using the relationship in Equation (8) for a $1 \mathrm{~km}$ horizontal path at $0.5 \mu \mathrm{m}$ wavelength, the $\mathrm{r}_{0}$ needs to be at least $1.26 \mathrm{~cm}$ in order for there to exist a tile size that will give nice speckle processed results. This now explains in a quantitative way why we have obtained good results in nearly all of our experiment; the $\mathrm{r}_{0}$ 's have been on the order of $1.5 \mathrm{~cm}$ to $3 \mathrm{~cm}$ for our near-range $(0.5 \mathrm{~km}$ to $3.5 \mathrm{~km})$ horizontal and low slant imaging paths. For the longer range $(20$ $\mathrm{km}$ to $60 \mathrm{~km}$ ) imaging experiments from tall hillsides with good results, similar $\mathrm{r}_{0}$ 's have been observed, but over higher slant paths with much of the turbulence well below the imaging path, indicating that Equation 8 is too strict, or that the effective atmospheric layer distance, $\mathrm{L}$, is quite near to the telescope.

\section{ACKNOWLEDGEMENTS}

I would like to thank Scot Olivier for allowing the use of his face in the simulations as well as the participation of LLNL security officer Goforth (Figure 14). Additionally, I'd like to thank my proofreaders - James Brase, Don Gavel, and Lisa Poyneer.

Work performed under the auspices of the U.S. Department of Energy by the University of California, Lawrence Livermore National Laboratory under contract No. W-7405-ENG-48.

\section{REFERENCES}

1. C. J. Carrano, "Speckle Imaging over Horizontal Paths", Proceedings of the SPIE -High Resolution Wavefront Control: Methods, Devices, and Applications IV, 4825, 109-120, (2002)

2. C. J. Carrano, J. M. Brase, "Horizontal and Slant Path Surveillance with Speckle Imaging", AMOS Technical 
Conference Proceedings, (2002)

3. C. J. Carrano, "Progress in horizontal and slant-path imaging using speckle imaging", Proceedings of the SPIELASE2003 - Optical Engineering at the Lawrence Livermore National Laboratory, 5001 (2003) pp. 56-64

4. T. J. Cornwell, "Radio-interferometric imaging of weak objects in conditions of poor phase stability: the relationship between speckle masking and phase closure methods", Astronomy and Astrophysics, 180, 269-274 (1987)

5. T. W. Lawrence, D. M. Goodman, E. M. Johansson, and J.P. Fitch, "Speckle imaging of satellites at the U.S. Air Force Maui Optical Station", Appl. Opt. 31, 6307-6321 (1992)

6. John W. Hardy, Adaptive Optics for Astronomical Telescopes, Oxford University Press (1998) pp. 102-103

7. Marechal, A., 1947, Rev. d'Optique 26, 257

8. J. Stone, P. H. Hu, S. P. Mills, and S. Ma, "Anisplanatic effects in finite-aperture optical systems", JOSA-A, Vol. 11, No. 1, pp. 347-357 (1994) 